\title{
Impact of peri-operative blood transfusion on post-operative infections after radical gastrectomy for gastric cancer: a propensity score matching analysis focusing on the timing, amount of transfusion and role of leukocyte depletion
}

\author{
Hua Xiao ${ }^{1} \cdot$ Hu Quan ${ }^{1}$. Shuguang Pan $^{1} \cdot$ Bin Yin $^{1} \cdot$ Wei Luo ${ }^{1} \cdot$ Gang Huang ${ }^{1} \cdot$ Yongzhong Ouyang ${ }^{1}$
}

Received: 19 January 2018 / Accepted: 20 March 2018 / Published online: 23 March 2018

(c) The Author(s) 2018

\begin{abstract}
Purpose Allogeneic blood transfusions (BTF) are sometimes inevitable during radical gastrectomy with lymphadenectomy for advanced gastric cancer. The aim of this retrospective study was to investigate the association between BTF and postoperative infections, focusing on the impact of timing, amount of transfusion and the role of leukocyte depletion.

Methods The study cohort was 2064 patients who underwent gastrectomy for gastric cancer from November 2010 to August 2017. The association between BTF and post-operative infections was estimated by univariate and multivariate analyses after propensity score matching. Subgroup analysis was performed according to the timing and amount of transfusion, and leukocyte depletion or not.

Results Out of a total 2064 patients, 426 (20.6\%) received peri-operative BTF. After one-to-one matching, 361 pairs of patients were included for further analysis, of who 68 (9.4\%) developed infections. Multivariate analysis identified that an operation time $\geq 240 \mathrm{~min}$, combined multi-organ resection, BTF and BMI $\geq 25 \mathrm{~kg} / \mathrm{m}^{2}$ were independent risk factors for post-operative infection. Patients given a high-volume (>7.5 U), intra-operatively of leukocyte-non-depleted BTF had the highest risk of developing infections clarified by subgroup analysis.

Conclusion Infection was the most common complication following gastrectomy for gastric cancer and BTF was identified as an independent risk factor by propensity score matching and multivariate analyses. The timing, amount of transfusion and leukocyte depletion had an impact on the incidence of infection. To decrease infection, BTF should be avoided where possible, particularly during operation, with a large amount and leukocyte-not-depleted blood.
\end{abstract}

Keywords Gastric cancer · Gastrectomy · Transfusion · Infection

\section{Introduction}

Gastric cancer is the fourth most frequent cancer occurring worldwide and the second most common cause of cancerrelated mortality in China (Torre et al. 2015; Chen et al. 2016), with to date surgery as the only treatment that offers

Hua Xiao and Hu Quan contributed equally to this work.

Yongzhong Ouyang

oyyz@vip.sina.com

1 Department of Gastroduodenal and Pancreatic Surgery, Hunan Cancer Hospital and the Affiliated Cancer Hospital of Xiangya School of Medicine, Central South University, 283 Tongzipo Road, Changsha 410013, Hunan, China a curative result. Unfortunately, the overwhelming majority of patients in China and Western countries are diagnosed at an advanced stage and radical gastrectomy with D2 lymph node dissection is recommended in the guidelines for these patients in the East and West (Japanese Gastric Cancer Association 2017; Ajani et al. 2016; Smyth et al. 2016). A high frequency of patients with advanced gastric cancer present with anemia, and furthermore, gastrectomy with lymphadenectomy sometimes causes excessive bleeding even when performed by experienced surgeons (Birgegård et al. 2005; Sasako et al. 2008). Thus, allogeneic blood transfusion (BTF) is sometimes inevitable when performing D2 gastrectomy for advanced gastric cancer, although it is fair to say that the frequency of BTF is decreasing as a result of improvements in surgical 
techniques and peri-operative care (Ecker et al. 2016). While BTF may be vital in some circumstances, there is growing evidence that BTF is associated with adverse long-term survival in oncological patients. These detrimental effects are thought to be associated with systemic inflammation and transfusion-related immunomodulation (TRIM) (Sun et al. 2015; Squires et al. 2015; Kanda et al. 2016; Aquina et al. 2017), while the relationships between BTF and post-operative short-term outcomes have been less well documented. Most of the current literature that reported investigations into the association between BTF and infection was usually based on a limited number of patients and none of these studies used propensity scorematching analysis to adjust for patient background data such as comorbidities, body mass index (BMI) or extension of resection, which are well-known risk factors for post-operative infection (Bellantone et al. 1998; Jung et al. 2013; Elmi et al. 2016). In addition, the association of the transfusion timing, amount and the role of leukocyte depletion with post-operative infection that focused on patients who underwent gastrectomy for gastric cancer has rarely been studied. Therefore, the present inquiry aimed to evaluate the potential impact of the timing, amount of transfusion and leukocyte depletion on post-operative infection after radical gastrectomy for gastric cancer by a propensity score-matching analysis using the database from a high volume center in China.

\section{Methods}

\section{Design and patients}

All consecutive adult patients ( $\geq 18$ years) who underwent surgery with a pathological diagnosed gastric adenocarcinoma between November 1, 2010 and August 31, 2017 in our hospital were screened for inclusion. Exclusion criteria are described in Fig. 1. In total, data from 2,064 patients were analyzed in this retrospective study. Patients were categorized according to whether they received peri-operative BTF or not. The ethics committee of the Affiliated Cancer Hospital of Xiangya School of Medicine, Central South University, approved this study and waived the need for informed consent considering the observational nature of the study design.

Fig. 1 Flow-chart

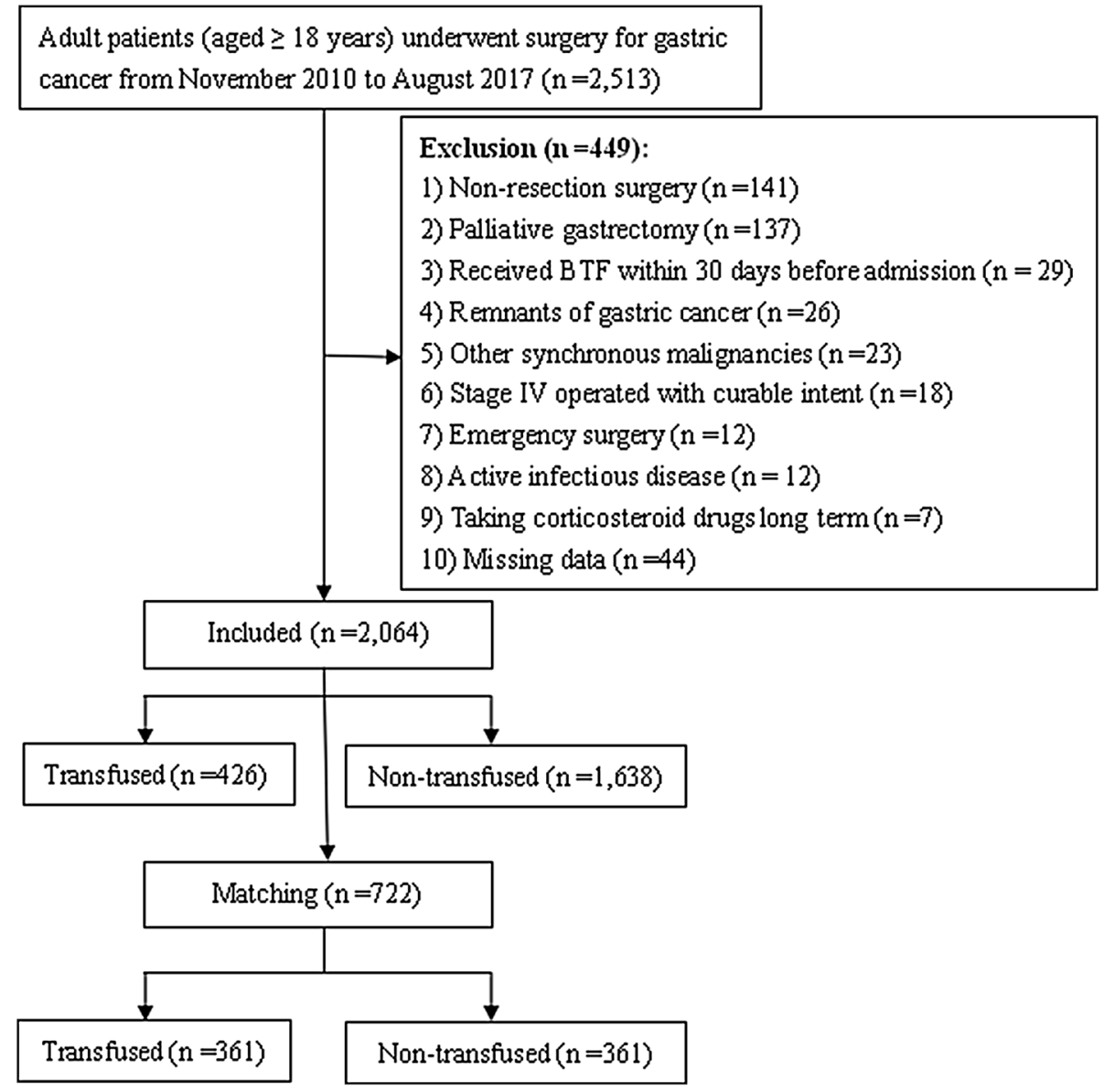




\section{Surgical procedures and peri-operative management}

Surgeons with sufficient experience of D2 or D2 + radical gastrectomy performed all operations. Each tumor was graded according to the 7th UICC (Union for International Cancer Control) TNM (Tumor-Lymph Node-Metastasis) Staging System of Gastric Cancer (Kwon 2011). Lymphadenectomy and gastric reconstruction were determined in accordance with Japanese gastric cancer treatment guidelines (Japanese Gastric Cancer Association 2017). The main surgical procedures and peri-operative management have been described in our previous study (Xiao et al. 2017). To be brief, open procedure with D2 or D2 + lymph node dissection was the main surgical type for patients with advanced gastric cancer. Combined multi-organ resection was performed in patients with a locally advanced tumor suspected of invading adjacent organs for the purpose of R0 resection or simultaneous resection of other organs because of benign disease. A 6-mm silicon drain tube was placed in the Morrison pouch and sub-hepatic space, and another placed in the splenic fossa if radical total gastrectomy or combined distal pancreatectomy and splenectomy was performed. A prophylactic antibiotic of a second- or third-generation cephalosporin was administered to all patients and usually lasted for 3-5 days following the operation. Neoadjuvant chemotherapy was applied in a few patients with advanced gastric cancer, in a standard manner with S-1 plus oxaliplatin (SOX), or epirubicin, cisplatin plus fluorouracil (ECF) as the main regimens for 2 to 4 cycles before surgery (Japanese Gastric Cancer Association 2017; Cunningham et al. 2006).

\section{Definition of BTF}

Peri-operative BTF was defined as the transfusion of red blood cells from the admission time to the day of discharge during hospitalization (usually 3-5 days before operation and 10-14 days thereafter). Packed red blood cells were stored in citrate-phosphate-dextrose-adenine anti-coagulant solution whether leukocytes were depleted or not. Although transfusion was performed at the discretion of the healthcare team supervising peri-operative care, the general indication for BTF was the hemoglobin level $<80 \mathrm{~g} / \mathrm{L}$. For patients with hemoglobin level between 80 and $100 \mathrm{~g} / \mathrm{L}, \mathrm{BTF}$ was performed based on the risk factors associated with inappropriate oxygenation or hemodynamic unstability (over 65 years, with cardiovascular or respiratory diseases, oxygen consumption, rate of blood loss, and so on) (American Society of Anesthesiologists Task Force on Perioperative Blood Transfusion and Adjuvant Therapies 2006).

The total amount of BTF was classified as low ( $<3 \mathrm{U}$, low-volume BTF group), moderate (3-7.5 U, moderate-volume BTF group) and high volume (>7.5 U, high-volume BTF group) (Bellantone et al. 1998). According to transfusion timing, BTF was classified into pre-operative (transfusion between admission and surgery, pre-operative BTF group), intra-operative (transfusion during surgery, intra-operative BTF group), and post-operative transfusion (transfusion between surgery and discharge, post-operative BTF group). For those patients who transfused more than one time described above, the first BTF timing was cited for classification.

The type of BTF was classified as leukocyte depleted or leukocyte non-depleted, according to the transfused blood type. The few patients who received both leukocyte-depleted and non-depleted red cells were assigned into the nondepleted BTF group.

\section{Definition of post-operative infections}

The responsible surgeon checked for the development of post-operative morbidity every day during the hospital stay and at every outpatient visit until 30 days after surgery, with morbidity classified into infectious and non-infectious complications. Patients were examined for the presence of any infection according to general surgical practice, clinical symptoms, temperature $\geq 38^{\circ} \mathrm{C}$ and/or increased inflammatory biochemical markers (white blood cell count). The diagnosis of different infectious complications (intra-abdominal infection, pneumonia, urinary tract infection, wound infection, and sepsis) was defined according to the Centers for Disease Control and Prevention (Horan and Andrus 2008). Briefly speaking, intra-abdominal infection was defined as an abscess or diffused infection within the abdominal cavity or the presence of an anastomotic leakage. Pneumonia was defined as an infection of the lung based on infiltrate on chest X-ray and/or computed tomography and clinical findings (respiratory symptoms, fever, leukopenia or leukocytosis). Sepsis was defined as the presence of two or more systemic inflammatory response syndrome criteria. Wound infection was defined as deep surgical site infection that required treatment with antibiotics agents or wound drainage. Urinary tract infection was defined according to positive urine culture and clinical findings (fever, urgency, frequency, dysuria, or suprapubic tenderness).

\section{Data collection}

The clinicopathological factors that could potentially influence the likelihood of post-operative outcomes were collected and analyzed. These factors included age, gender, American Society of Anesthesiologists (ASA) score, body mass index (BMI), comorbidities (diabetes mellitus, hypertension, chronic pulmonary/kidney/liver disease, cardiovascular and cerebrovascular disease), pre-operative white 
blood cell and lymphocyte counts, pre-operative albumin and hemoglobin levels, the type of gastrectomy, operation times, estimated intra-operative blood loss, the pathological TNM stage and so on. Morbidity and mortality data were collected within 30 days post-operatively and classified in accordance with the modified Clavien-Dindo classification (Dindo et al. 2004). Major complications were defined as Clavien-Dindo grade III or greater. For those patients who developed multiple complications, Clavien-Dindo stage was classified according to the most severe one.

\section{Matching}

To minimize the impact of potential selective bias, transfused patients were matched to non-BTF patients using a propensity score described by Rubin et al. (Rubin and Thomas 1996), and was done as previously described (Yang et al. 2016). Propensity scores were based on baseline variables such as age, sex, comorbidities, ASA score, neoadjuvant chemotherapy, pre-operative white blood cell and lymphocyte count, pre-operative albumin level, operation method, type of resection, combined multi-organ resection, tumor size, tumor location, operation time and the TNM stage. Nearest neighbor matching was performed in a oneto-one ratio without replacement and a caliper width with a 0.01 standard deviation (SD) was specified.

\section{Statistical analysis}

Statistical analyses were performed with IBM SPSS Statistics for Windows (Ver. 24, NY: IBM Corporation). Continuous data are reported as the mean \pm SD and comparisons made on normally distributed data using a $t$ test. Categorical variables are expressed as numbers and percentages, and were compared by a $\chi^{2}$ or Fisher exact test. While for ordinal categorical variables, the differences between groups were compared by a rank sum test. Subgroup analysis was performed according to the transfusion timing, amount and leukocyte depletion or not. Risk factors for infection were subjected to univariate analyses using a $\chi^{2}$ test to assess the effects of each factor. Multivariate logistic regression analysis was carried out for factors with a $P$ value $\leq 0.1$ after univariate analysis. A $P$ value $<0.05$ was considered to represent statistical significance.

\section{Results}

\section{Clinicopathological characteristics of the patients}

The clinicopathological characteristics of the 2064 patients are listed in Table 1. There were 1363 males $(66.0 \%)$ and $701(34.0 \%)$ females, with a mean age of $55.37 \pm 10.51$ years (range 19-83) and a mean BMI of $21.80 \pm 2.97 \mathrm{~kg} / \mathrm{m}^{2}$ (range 13.84-35.17). 622 patients (30.1\%) suffered from one or more types of comorbidities. A total gastrectomy was performed on 482 patients (23.4\%), distal subtotal gastrectomy on $1523(73.8 \%)$ patients, and proximal subtotal gastrectomy on 59 patients (2.9\%). Laparoscopic or laparoscopy-assisted procedures were performed on 307 patients (14.9\%), and 184 patients $(8.9 \%)$ underwent combined multi-organ resection, including 47 cases of cholecystectomy, 45 cases of partial pancreatectomy, 31 cases of splenectomy and so on. According to the 7th edition of the UICC TNM classification, there were $528(25.6 \%)$ stage I, $434(21.0 \%)$ stage II, and 1102 (53.4\%) stage III patients. The mean operation time was $200 \pm 54$ min (range 70-584), and the mean estimated intraoperative blood loss was $205 \pm 120 \mathrm{~mL}$ (range 30-2300).

426 patients $(20.6 \%)$ underwent peri-operative BTF and the median amount of blood transfusion was $4 \mathrm{U}$ (range 1.5-42.5). As shown in Table 1, patients receiving BTF were obviously older, with lower pre-operative hemoglobin levels, higher rates of complications due to the tumor (including pyloric obstruction or bleeding), total gastrectomy and/or combined multi-organ resection were performed, at stage III, and had larger tumor sizes and longer operation times.

\section{Post-operative complications}

A total of 376 events occurred in 323 patients of the entire cohort $(15.6 \%)$. There were 156 events that were classified as grade I complications which occurred in 141 patients (6.8\%), including transient vomiting, fever, fluid collection, and/or pain needing antiemetics, antipyretics, diuretics, and/or analgesics to relieve the symptoms $(n=94)$, wound problem treated in bedside $(n=31)$, atelectasis requiring physiotherapy $(n=18)$, and bladder dysfunction requiring urinary catheterization $(n=13)$. Considering their little clinical relevance, grade I complications were not included for further analysis, as reported by Ahmad et al. (2014). The remaining 220 post-operative grade II or greater complications occurred in 182 patients (8.8\%), including 139 (63.2\%) infections and 81 (36.8\%) non-infection complications (Table 2). Among the infection complications, intra-abdominal infection $(n=74)$ was the most common, followed by pneumonia $(n=51)$ and sepsis $(n=15)$. While intestinal obstruction $(n=15)$, pleural effusion $(n=12)$ and ascites $(n=11)$ were ranked as the top three most frequent noninfection complications. According to the Clavien-Dindo classification system, the incidence of stage II, IIIa, IIIb, IVa, IVb and V complications were $6.1 \%(n=125), 0.9 \%$ $(n=19), 0.9 \%(n=19), 0.4 \%(n=9), 0.05 \%(n=1)$ and $0.4 \%$ $(n=9)$, respectively. Thus major complications occurred in 57 patients $(2.8 \%) .73$ patients $(17.1 \%)$ in the BTF group developed complications, which were significantly more 
Table 1 Clinicopathological characteristics of the entire cohort $(n=2064)$

\begin{tabular}{|c|c|c|c|}
\hline Variables & $\begin{array}{l}\text { Transfusion group } \\
(n=426)\end{array}$ & $\begin{array}{l}\text { Non-transfusion group } \\
(n=1638)\end{array}$ & $P$ value \\
\hline Gender (males) & $267(62.3 \%)$ & $1,096(66.9 \%)$ & 0.100 \\
\hline Age (years) & $57.91 \pm 11.17$ & $54.72 \pm 10.24$ & $<0.001$ \\
\hline Body Mass Index $\left(\mathrm{kg} / \mathrm{m}^{2}\right)$ & $21.95 \pm 3.01$ & $21.24 \pm 2.78$ & $<0.001$ \\
\hline ASA score & & & $<0.001$ \\
\hline 1 & $53(12.4 \%)$ & $261(15.9 \%)$ & \\
\hline 2 & $286(67.1 \%)$ & $1224(74.7 \%)$ & \\
\hline 3 & $83(19.5 \%)$ & $147(9.0 \%)$ & \\
\hline 4 & $4(0.9 \%)$ & $6(0.4 \%)$ & \\
\hline Smoking history & $164(38.5 \%)$ & $717(43.8 \%)$ & 0.050 \\
\hline Any comorbidities & $145(34.0 \%)$ & $477(29.1 \%)$ & 0.049 \\
\hline History of abdominal surgery & $49(11.5 \%)$ & $163(10.0 \%)$ & 0.347 \\
\hline Neo-adjuvant chemotherapy & $27(6.3 \%)$ & $98(6.0 \%)$ & 0.784 \\
\hline Pre-operative white blood cell count $\left(\times 10^{9} / \mathrm{L}\right)$ & $5.90 \pm 2.00$ & $6.21 \pm 1.83$ & 0.002 \\
\hline Pre-operative lymphocyte count $\left(\times 10^{9} / \mathrm{L}\right)$ & $1.56 \pm 0.77$ & $1.86 \pm 0.64$ & $<0.001$ \\
\hline Pre-operative albumin (g/L) & $35.83 \pm 4.89$ & $38.81 \pm 4.28$ & $<0.001$ \\
\hline Pre-operative hemoglobin (g/L) & $89.62 \pm 24.11$ & $126.23 \pm 18.26$ & $<0.001$ \\
\hline Complication due to the tumor ${ }^{\mathrm{a}}$ & $203(47.7 \%)$ & $252(15.4 \%)$ & $<0.001$ \\
\hline Operation method & & & 0.008 \\
\hline Open & $380(89.2 \%)$ & $1377(84.1 \%)$ & \\
\hline Laparoscopy & $46(10.8 \%)$ & $261(15.9 \%)$ & \\
\hline Type of resection & & & $<0.001$ \\
\hline Subtotal gastrectomy & $290(68.1 \%)$ & $1292(78.9 \%)$ & \\
\hline Total gastrectomy & $136(31.9 \%)$ & $346(21.1 \%)$ & \\
\hline Combined multi-organ resection & $77(18.1 \%)$ & $107(6.5 \%)$ & $<0.001$ \\
\hline Tumor size $(\mathrm{cm})$ & $4.94 \pm 2.11$ & $3.89 \pm 1.99$ & $<0.001$ \\
\hline Tumor location & & & $<0.001$ \\
\hline Upper & $53(12.4 \%)$ & $123(7.5 \%)$ & \\
\hline Middle & $114(26.8 \%)$ & $316(19.3 \%)$ & \\
\hline Lower & $237(55.6 \%)$ & $1148(70.1 \%)$ & \\
\hline Diffuse & $22(5.2 \%)$ & $51(3.1 \%)$ & \\
\hline pTNM stage $^{\mathrm{b}}$ & & & $<0.001$ \\
\hline I & $57(13.4 \%)$ & $471(28.8 \%)$ & \\
\hline II & $66(15.5 \%)$ & $368(22.5 \%)$ & \\
\hline III & $303(71.1 \%)$ & $799(48.8 \%)$ & \\
\hline Intra-operative blood loss (mL) & $261 \pm 200$ & $190 \pm 83$ & $<0.001$ \\
\hline Operation time (min) & $215.75 \pm 62.13$ & $196.22 \pm 50.87$ & $<0.001$ \\
\hline Post-operative complications classification & & & $<0.001$ \\
\hline None & $353(82.9 \%)$ & $1,529(93.3 \%)$ & \\
\hline Infectious complications & $51(12.0 \%)$ & $78(4.8 \%)$ & \\
\hline Non-infectious complications & $22(5.2 \%)$ & $31(1.9 \%)$ & \\
\hline Post-operative complications severity ${ }^{\mathrm{c}}$ & & & $<0.001$ \\
\hline None & $353(82.9 \%)$ & $1529(93.3 \%)$ & \\
\hline Grade II & $45(10.6 \%)$ & $80(4.9 \%)$ & \\
\hline Grade III or greater & $28(6.6 \%)$ & $29(1.8 \%)$ & \\
\hline Transferring to ICU post-operation & $22(5.2 \%)$ & $27(1.6 \%)$ & $<0.001$ \\
\hline Post-operative hospital stays (days) & $13.47 \pm 11.87$ & $11.37 \pm 3.70$ & $<0.001$ \\
\hline
\end{tabular}

Data are presented as mean \pm SD or $n(\%)$

ASA American Society of Anesthesiology, ICU intensive care unit

${ }^{a}$ Including pyloric obstruction or bleeding

${ }^{\mathrm{b}}$ Tumor stages are based on 7th edition of the Union for International Cancer Control TNM classification

${ }^{\mathrm{c}}$ Based on the Clavien-Dindo severity classification of surgical complications 
Table 2 Post-operative complications of the entire 2064 patients $(n=220)$

\begin{tabular}{lc}
\hline Complications & Number $(\%)$ \\
\hline Infectious & $139(63.2 \%)$ \\
Intra-abdominal infection & $74(33.6 \%)$ \\
Pneumonia & $39(17.7 \%)$ \\
Sepsis not specified & $15(6.8 \%)$ \\
Wound infection & $9(4.1 \%)$ \\
Urinary tract infection & $2(0.9 \%)$ \\
Non-infectious & $81(36.8 \%)$ \\
Intestinal obstruction & $15(6.8 \%)$ \\
Pleural effusion & $12(5.5 \%)$ \\
Ascites & $11(5.0 \%)$ \\
Intra-abdominal bleeding & $10(4.5 \%)$ \\
Lymphatic fistula & $7(3.2 \%)$ \\
Gastrointestinal bleeding & $7(3.2 \%)$ \\
Duodenal stump fistula & $3(1.4 \%)$ \\
Cerebral infarction & $3(1.4 \%)$ \\
Delayed gastric emptying & $2(0.9 \%)$ \\
Anastomotic stricture & $2(0.9 \%)$ \\
Liver failure & $2(0.9 \%)$ \\
Pneumothorax & $2(0.9 \%)$ \\
Cardiac arrest & $2(0.9 \%)$ \\
Diabetic ketoacidosis & $1(0.5 \%)$ \\
Urinary retention & $1(0.5 \%)$ \\
Renal failure & $1(0.5 \%)$ \\
\hline
\end{tabular}

common than that in the non-BTF group $(6.7 \%, P<0.001)$, as was the infection rate $(12.0 \%$ vs $4.8 \%, P<0.001)$.

\section{Propensity score matching analysis}

After one-to-one propensity score matching, 361 pairs of patients were enrolled into further analysis. The clinicopathological characteristics of the patients after matching are listed in Table 3. All of the important basic characteristics such as comorbidities, pre-operative white blood cell/ lymphocyte counts, the tumor stage and operation times were comparable between the two groups, except for the pre-operative hemoglobin levels $(91.19 \pm 23.99 \mathrm{~g} / \mathrm{L}$ vs . $119.98 \pm 17.38 \mathrm{~g} / \mathrm{L}, P<0.001)$ and estimated intra-operative blood loss $(248 \pm 177 \mathrm{~mL}$ vs. $212 \pm 90 \mathrm{~mL}, P=0.001)$. Sixty patients $(16.6 \%)$ in the BTF group developed post-operative complications, including 43 cases (11.9\%) of infection, which was significantly higher than those in the non-BTF group (9.7\% and 6.9\%, $P=0.006$ and $P=0.022$, respectively).

\section{Subgroup analysis}

Of the 361 patients receiving BTF who were enrolled into further analysis after matching, the median amount of transfused blood was $4 \mathrm{U}$ (range 1.5-42.5). The transfusion timing is shown in detail in Table 4a. There were 149 patients who received transfusion pre-operatively, 128 intraoperatively and 189 post-operatively. The incidence of infection in the intra-operative BTF group was $14.1 \%$, and was significantly higher than in the non-BTF and post-operative BTF groups ( 6.9 and $6.9 \%, P=0.014$ and $P=0.035$, respectively). However, the infection rates among the non-BTF, pre-operative and post-operative BTF groups were not statistically different. There were 95 patients who received BTF more than once in the period described above, of whom 15 (15.8\%) developed infections, which was significantly higher than that in the non-BTF group $(6.9 \%, P=0.007)$, but comparable with those in the only one time period transfused patients $(10.5 \%, P=0.174)$.

74 patients $(20.5 \%)$ received a low-volume $(<3 \mathrm{U}), 228$ $(63.2 \%)$ a moderate-volume (3-7.5 U), and $59(16.3 \%)$ a high-volume (>7.5 U) BTF, respectively (Table 4b). 12 patients $(20.3 \%)$ in the high-volume BTF group suffered from post-operative infections, which were significantly higher than those in the non-BTF group $(6.9 \%, P=0.001)$, and moderate-volume BTF group $(10.1 \%, P=0.032)$. In contrast, the infection rates among the non-BTF, low-volume and moderate-volume BTF groups were not significantly different.

221 patients $(61.2 \%)$ received BTF with leukocyte depletion and the remaining 140 were transfused with leukocytenon-depleted blood (Table 4c). Patients receiving leukocytenon-depleted BTF had a higher risk of infection compared with those not receiving BTF (13.6 and 6.9\%, $P=0.018$ ). Patients who were transfused with leukocyte-depleted blood appeared to have a trend toward a higher incidence of infection compared with those not receiving BTF (10.9 vs $6.9 \%)$, but the difference was not statistically significant $(P=0.097)$. There was no significant difference between the incidence of infections among patients who received BTF with leukocyte-depleted or non-depleted blood $(P=0.438)$.

\section{Univariate and multivariate logistic regression analysis}

On univariate analysis (Table 5), combined multi-organ resection, operation time $\geq 240 \mathrm{~min}, \mathrm{BMI} \geq 25 \mathrm{~kg} / \mathrm{m}^{2}$, a history of smoking, splenectomy and BTF were clarified as risk factors for infection in the 722 matched patients. After multivariate analysis (Table 6), including factors that had $P$ values $\leq 0.1$ established by univariate analysis, operation time $\geq 240 \mathrm{~min}$ (odds ratio, $\mathrm{OR}=2.378,95 \%$ confidence interval, CI 1.393-4.062, $P=0.002$ ), combined multi-organ resection $(\mathrm{OR}=2.418,95 \%$ CI $1.281-4.561, P=0.006)$, BTF $(\mathrm{OR}=1.872,95 \%$ CI $1.094-3.204, P=0.022)$ and $\mathrm{BMI} \geq 25 \mathrm{~kg} / \mathrm{m}^{2} \quad(\mathrm{OR}=2.149,95 \%$ CI $1.074-4.299$, $P=0.031)$ were shown to be independent risk factors for 
Table 3 Clinicopathological characteristics of the propensity score-matched cohort $(n=722)$

\begin{tabular}{|c|c|c|c|}
\hline Variables & $\begin{array}{l}\text { Transfusion group } \\
(n=361)\end{array}$ & $\begin{array}{l}\text { Non-transfusion group } \\
(n=361)\end{array}$ & $P$ value \\
\hline Gender (males) & $225(62.3 \%)$ & $229(63.4 \%)$ & 0.758 \\
\hline Age (years) & $57.15 \pm 10.92$ & $55.76 \pm 11.43$ & 0.096 \\
\hline Body Mass Index $\left(\mathrm{kg} / \mathrm{m}^{2}\right)$ & $21.26 \pm 2.74$ & $21.24 \pm 2.77$ & 0.895 \\
\hline ASA score & & & 0.067 \\
\hline 1 & $48(13.3 \%)$ & $46(12.7 \%)$ & \\
\hline 2 & $256(70.9 \%)$ & $233(64.5 \%)$ & \\
\hline 3 & $54(15.0 \%)$ & $77(21.3 \%)$ & \\
\hline 4 & $3(0.8 \%)$ & $5(1.4 \%)$ & \\
\hline Smoking history & $136(37.7 \%)$ & $125(34.6 \%)$ & 0.394 \\
\hline Any comorbidities & $115(31.9 \%)$ & $119(33.0 \%)$ & 0.750 \\
\hline History of abdominal surgery & $40(11.1 \%)$ & $37(10.2 \%)$ & 0.718 \\
\hline Neo-adjuvant chemotherapy & $26(7.2 \%)$ & $16(4.4 \%)$ & 0.112 \\
\hline Pre-operative white blood cell count $\left(\times 10^{9} / \mathrm{L}\right)$ & $5.86 \pm 1.87$ & $6.10 \pm 1.73$ & 0.080 \\
\hline Pre-operative lymphocyte count $\left(\times 10^{9} / \mathrm{L}\right)$ & $1.62 \pm 0.74$ & $1.66 \pm 0.65$ & 0.416 \\
\hline Pre-operative albumin $(\mathrm{g} / \mathrm{L})$ & $36.39 \pm 4.87$ & $36.91 \pm 4.96$ & 0.154 \\
\hline Pre-operative hemoglobin $(\mathrm{g} / \mathrm{L})$ & $91.19 \pm 23.99$ & $119.98 \pm 17.38$ & $<0.001$ \\
\hline Complication due to the tumor ${ }^{\mathrm{a}}$ & $147(40.7 \%)$ & $147(40.7 \%)$ & 1.000 \\
\hline Operation method & & & 0.091 \\
\hline Open & $322(89.2 \%)$ & $335(92.8 \%)$ & \\
\hline Laparoscopy & $39(10.8 \%)$ & $26(7.2 \%)$ & \\
\hline Type of resection & & & 0.203 \\
\hline Subtotal gastrectomy & $260(72.0 \%)$ & $275(76.2 \%)$ & \\
\hline Total gastrectomy & $101(28.0 \%)$ & $86(23.8 \%)$ & \\
\hline Combined multi-organ resection & $52(14.4 \%)$ & $50(13.9 \%)$ & 0.831 \\
\hline Tumor size $(\mathrm{cm})$ & $4.77 \pm 2.11$ & $4.86 \pm 2.02$ & 0.544 \\
\hline Tumor location & & & 0.441 \\
\hline Upper & $43(11.9 \%)$ & $40(11.1 \%)$ & \\
\hline Middle & $92(25.5 \%)$ & $75(20.8 \%)$ & \\
\hline Lower & $210(58.2 \%)$ & $228(63.2 \%)$ & \\
\hline Diffuse & $16(4.4 \%)$ & $18(5.0 \%)$ & \\
\hline pTNM stage $^{\mathrm{b}}$ & & & 0.977 \\
\hline I & $55(15.2 \%)$ & $43(11.9 \%)$ & \\
\hline II & $58(16.1 \%)$ & $74(20.5 \%)$ & \\
\hline III & $248(68.7 \%)$ & $244(67.6 \%)$ & \\
\hline Intra-operative blood loss (mL) & $248 \pm 177$ & $212 \pm 90$ & 0.001 \\
\hline Operation time $(\mathrm{min})$ & $211.78 \pm 58.61$ & $216.61 \pm 45.50$ & 0.199 \\
\hline Post-operative complication classification & & & 0.023 \\
\hline None & $301(83.4 \%)$ & $326(90.3 \%)$ & \\
\hline Infectious complications & $43(11.9 \%)$ & $25(6.9 \%)$ & \\
\hline Non-infectious complications & $17(4.7 \%)$ & $10(2.8 \%)$ & \\
\hline Post-operative complication severity ${ }^{c}$ & & & 0.006 \\
\hline None & $301(83.4 \%)$ & $326(90.3 \%)$ & \\
\hline Grade II & $38(10.5 \%)$ & $23(6.4 \%)$ & \\
\hline Grade III or greater & $22(6.1 \%)$ & $12(3.3 \%)$ & \\
\hline Transferring to ICU post-operation & $16(4.4 \%)$ & $11(3.0 \%)$ & 0.327 \\
\hline Post-operative hospital stays (days) & $13.20 \pm 10.70$ & $12.34 \pm 4.05$ & 0.153 \\
\hline
\end{tabular}

Data are presented as mean $\pm \mathrm{SD}$ or $n(\%)$

ASA American Society of Anesthesiology, ICU intensive care unit

${ }^{a}$ Including pyloric obstruction or bleeding

${ }^{\mathrm{b}}$ Tumor stages are based on 7th edition of the Union for International Cancer Control TNM classification

${ }^{c}$ Based on the Clavien-Dindo severity classification of surgical complications 
Table 4 Subgroup analysis after matching $(n=722)$

\begin{tabular}{|c|c|c|}
\hline \multirow{2}{*}{$\begin{array}{l}\text { Variables } \\
\text { Transfusion timing }\end{array}$} & \multicolumn{2}{|l|}{ Complications } \\
\hline & None & Infections \\
\hline \multicolumn{3}{|l|}{ a. Transfusion timing and infections } \\
\hline Non-BTF group $(n=361)$ & $336(93.1 \%)$ & $25(6.9 \%)$ \\
\hline Pre-operative BTF group $(n=149)$ & $136(91.3 \%)$ & $13(8.7 \%)$ \\
\hline Intra-operative BTF group $(n=128)$ & $110(85.9 \%)$ & $18(14.1 \%)$ \\
\hline Post-operative BTF group $(n=189)$ & $176(93.1 \%)$ & $13(6.9 \%)$ \\
\hline One time-period BTF group $(n=266)$ & $238(89.5 \%)$ & $28(10.5 \%)$ \\
\hline Multi-time-period BTF group $(n=95)$ & $80(84.2 \%)$ & $15(15.8 \%)$ \\
\hline \multicolumn{3}{|c|}{$\begin{array}{l}\text { Comparison between subgroups: non-BTF vs. pre-operative } \mathrm{BTF}, P=0.482 \text {; non-BTF vs. intra-operative } \mathrm{BTF}, \boldsymbol{P}=\mathbf{0 . 0 1 4} \text {; non-BTF vs. } \\
\text { post-operative } \mathrm{BTF}, P=0.984 \text {; pre-operative } \mathrm{BTF} \text { vs. intra-operative } \mathrm{BTF}, P=0.160 \text {; pre-operative } \mathrm{BTF} \text { vs. post-operative } \mathrm{BTF}, P=0.527 \text {; } \\
\text { intra-operative BTF vs. post-operative } \mathrm{BTF}, \boldsymbol{P}=\mathbf{0 . 0 3 5}\end{array}$} \\
\hline \multicolumn{3}{|c|}{$\begin{array}{l}\text { Non-BTF vs. one time-period BTF, } P=0.109 \text {; non-BTF vs. multi-time-period BTF, } \boldsymbol{P}=\mathbf{0 . 0 0 7} \text {; one time-period BTF vs. multi-time-period } \\
\text { BTF, } P=0.174\end{array}$} \\
\hline \multirow{2}{*}{$\begin{array}{l}\text { Variables } \\
\text { Transfusion volume }\end{array}$} & \multicolumn{2}{|l|}{ Complications } \\
\hline & None & Infections \\
\hline \multicolumn{3}{|l|}{ b. Transfusion volume and infections } \\
\hline Non-BTF group $(n=361)$ & $336(93.1 \%)$ & $25(6.9 \%)$ \\
\hline Low-volume BTF group $(n=74)$ & $66(89.2 \%)$ & $8(10.8 \%)$ \\
\hline Moderate-volume BTF group $(n=228)$ & $205(89.9 \%)$ & $23(10.1 \%)$ \\
\hline High-volume BTF group $(n=59)$ & $47(79.7 \%)$ & $12(20.3 \%)$ \\
\hline \multicolumn{3}{|c|}{$\begin{array}{l}\text { Comparison between subgroups: non-BTF vs. low-volume BTF, } P=0.250 \text {; non-BTF vs. moderate-volume } \mathrm{BTF}, P=0.172 \text {; non-BTF vs. } \\
\text { high-volume } \mathrm{BTF}, \boldsymbol{P}=\mathbf{0 . 0 0 1} \text {; low-volume } \mathrm{BTF} \text { vs. moderate-volume } \mathrm{BTF}, P=0.859 \text {; low-volume } \mathrm{BTF} \text { vs. high-volume } \mathrm{BTF}, P=0.127 \text {; } \\
\text { moderate-volume BTF vs. high-volume BTF, } \boldsymbol{P}=\mathbf{0 . 0 3 2}\end{array}$} \\
\hline \multirow{2}{*}{$\begin{array}{l}\text { Variables } \\
\text { Transfusion type }\end{array}$} & \multicolumn{2}{|c|}{ Complications } \\
\hline & None & Infections \\
\hline \multicolumn{3}{|l|}{ c. Leukocyte depletion and infections } \\
\hline Non-BTF group $(n=361)$ & $336(93.1 \%)$ & $25(6.9 \%)$ \\
\hline Leukocyte-depleted BTF group $(n=221)$ & $197(89.1 \%)$ & $24(10.9 \%)$ \\
\hline Leukocyte-non-depleted BTF group $(n=140)$ & $121(86.4 \%)$ & $19(13.6 \%)$ \\
\hline \multicolumn{3}{|l|}{$B T F$ blood transfusion } \\
\hline \multicolumn{3}{|c|}{$\begin{array}{l}\text { Comparison between subgroups: non-BTF vs. leukocyte-depleted BTF, } P=0.097 \text {; non-BTF vs. leukocyte-non-depleted BTF, } P=\mathbf{0 . 0 1 8} \text {; } \\
\text { leukocyte-depleted BTF vs. leukocyte-non-depleted BTF, } P=0.438\end{array}$} \\
\hline
\end{tabular}

Statistically significant values are in bold $(p<0.05)$

infection. Smoking appeared to influence the incidence of infection ( $\mathrm{OR}=1.597,95 \%$ CI 0.945-2.699), but the finding was not statistically significant $(P=0.080)$. No other variables such as comorbidities, total gastrectomy or splenectomy were identified as independent risk factors for infection.

\section{Discussion}

In this retrospective study of a large cohort of patients from a single high-volume center in China, after one-to-one propensity score matching we found that infection was the most common complication following radical gastrectomy for gastric cancer, leading to prolonged post-operative hospital stays (23.78 vs. 11.62 days, $P<0.001)$ and a higher frequency of requiring intensive care (17.6 vs $2.3 \%$, $P<0.001)$ and also mortality (8.8 vs $0.2 \%, P<0.001)$. Thus, surgeons should prioritize operating procedures to reduce the incidence of infection. Although many studies have evaluated the impact of peri-operative BTF on postoperative outcomes of patients undergoing gastrectomy for gastric cancer, unfortunately most of the conclusions were based on a limited number of patients and unmatched analysis. Thus, the conclusions may be confused by other factors such as comorbidities and more extended resections. Propensity score matching analysis is widely used in retrospective cohort studies to control for confounding biases, mimicking a randomized trial, with the assumption 
Table 5 Univariate analysis of possible predictors for postoperative infections following radical gastrectomy for gastric cancer after matching $(n=722)$
Table 6 Multivariate analysis of possible predictors for postoperative infections following gastrectomy for gastric cancer after matching $(n=722)$

\begin{tabular}{lllrr}
\hline Variables & $\begin{array}{l}\text { Infections } \\
(n=68)\end{array}$ & $\begin{array}{l}\text { Non-infection } \\
(n=654)\end{array}$ & $\chi^{2}$ value & $P$ value \\
\hline Sex (male: female) & $48 / 20$ & $406 / 248$ & 1.911 & 0.167 \\
Age $($ years $) \geq 65 /<65$ & $20 / 48$ & $158 / 496$ & 0.915 & 0.339 \\
BMI $\left(\mathrm{kg} / \mathrm{m}^{2}\right) \geq 25 /<25$ & $13 / 55$ & $60 / 594$ & 6.701 & 0.010 \\
Smoking history; yes/no & $34 / 34$ & $227 / 427$ & 6.239 & 0.012 \\
ASA score $\geq 3 /<3$ & $16 / 52$ & $123 / 531$ & 0.883 & 0.347 \\
Comorbidity; yes/no & $28 / 40$ & $206 / 448$ & 2.634 & 0.105 \\
Pre-operative white blood cell count $\left(\times 10^{9} / \mathrm{L}\right) \geq 4 /<4$ & $64 / 4$ & $584 / 70$ & 1.556 & 0.212 \\
Pre-operative lymphocyte count $\left(\times 10^{9} / \mathrm{L}\right) \geq 1.5 /<1.5$ & $38 / 30$ & $353 / 301$ & 0.090 & 0.764 \\
Pre-operative hemoglobin $(\mathrm{g} / \mathrm{L}) \geq 100 /<100$ & $43 / 25$ & $389 / 265$ & 0.361 & 0.548 \\
Pre-operative albumin $(\mathrm{g} / \mathrm{L}) \geq 35 /<35$ & $39 / 29$ & $429 / 225$ & 1.836 & 0.175 \\
Complication due to the tumor ; yes/no & $28 / 40$ & $266 / 388$ & 0.006 & 0.936 \\
Neo-adjuvant chemotherapy; yes/no & $4 / 64$ & $38 / 616$ & 0.001 & 0.981 \\
Operation method; open/laparoscopy & $7 / 61$ & $58 / 596$ & 0.153 & 0.696 \\
Extent of gastric resection; subtotal/total & $48 / 20$ & $487 / 167$ & 0.482 & 0.487 \\
Combined multi-organ resection; yes/no & $22 / 46$ & $80 / 574$ & 20.555 & $<0.001$ \\
Splenectomy; yes/no & $5 / 63$ & $11 / 643$ & 0.141 & 0.002 \\
Operation time (min) $\geq 240 /<240$ & $36 / 32$ & $183 / 471$ & 18.159 & $<0.001$ \\
Intra-operative blood loss (mL) $\geq 300 /<300$ & $26 / 42$ & $191 / 463$ & 2.389 & 0.122 \\
Tumor size (cm) $\geq 5 /<5$ & $39 / 29$ & $343 / 311$ & 0.595 & 0.440 \\
Depth of invasion; T4/T1-3 & $55 / 13$ & $489 / 165$ & 1.239 & 0.266 \\
Lymph node metastasis; positive/negative & $48 / 20$ & $469 / 185$ & 0.038 & 0.845 \\
pTNM stage; III/I-II & $49 / 19$ & $433 / 221$ & 0.950 & 0.330 \\
Peri-operative BTF; yes/no & $43 / 25$ & $318 / 336$ & 5.260 & 0.022 \\
\hline & & &
\end{tabular}

$B M I$ body mass index, ASA American Society of Anesthesiologist, BTF blood transfusion

${ }^{a}$ Including pyloric obstruction or bleeding

\begin{tabular}{llll}
\hline Variables & Odds ratio (OR) & $\begin{array}{l}95 \% \text { confidence interval } \\
(\mathrm{CI})\end{array}$ & $P$ value \\
\hline Operation time $\geq 240$ min & 2.378 & $1.393-4.062$ & 0.002 \\
Combined multi-organ resection & 2.418 & $1.281-4.561$ & 0.006 \\
Peri-operative blood transfusion & 1.872 & $1.094-3.204$ & 0.022 \\
Body mass index $\geq 25 \mathrm{~kg} / \mathrm{m}^{2}$ & 2.149 & $1.074-4.299$ & 0.031 \\
Smoking history & 1.597 & $0.945-2.699$ & 0.080 \\
Splenectomy & 1.555 & $0.446-5.418$ & 0.480 \\
\hline
\end{tabular}

that all related confounders are controlled (Fujiya et al. 2016). As listed in Table 1, background data between the patients receiving BTF or not were significantly different before matching and thus it could be argued that direct comparison of the infection rate is not appropriate because some of the basic data could be independently responsible for infections, regardless of BTF. To the best of our knowledge, this is the first study to investigate the relationship between BTF and post-operative infections that has used propensity score-matching analysis to adjust for basic data between patients with or without BTF following gastrectomy for gastric cancer. After matching, most of the important background data become comparable except for pre-operative hemoglobin levels and intra-operative blood loss, which were considered to be the main factors associated with BTF. These data were not used for enrolment for matching to avoid too many patients who received BTF being excluded because of a lack of matching. Further univariate and multivariate analyses showed that pre-operative hemoglobin anemia $(<100 \mathrm{~g} / \mathrm{L})$ and intra-operative blood loss $\geq 300 \mathrm{~mL}$ were not associated with increased risk of post-operative infection.

The significance of the transfusion timing and volume, and impact of leukocyte depletion on post-operative 
infections have rarely been reported. By sub-group analysis, patients who received intra-operative BTF were identified as being at the highest risk of developing an infection, while pre-operative and post-operative BTF showed less impact. Possible explanations include that intra-operative BTF is usually performed because of massive bleeding, even hemorrhagic shock in patients with extended resection or iatrogenic injury, which may have increased the risk of infection. Intra-operative BTF may also act synergistically with surgical stress to induce immunosuppression (Kanda et al. 2016). Thus, for patients who suffered from anemia at admission, performing BTF pre-operatively instead of intra-operatively may decrease the risk of infection. Additionally, for patients with mild to moderate bleeding during surgery, given good hemodynamic stability, there could be some room for consideration of giving BTF post-operatively instead of intra-operatively. But the most suitable time interval between surgery and BTF is hard to define and requires further prospective investigation. Another interesting finding was that patients who received BTF in more than one time period, classified as pre-, intra- and post-operative, had a significantly higher risk of developing infection compared with those receiving BTF for only one time period, reminding us that we should avoid performing BTF in multi-time periods if possible. The potential cause may be because of the prolonged process of immunosuppression induced by BTF but the exact reasons remain to be elucidated.

The volume of BTF on post-operative outcomes remains controversial; some studies clarified that intra-operative transfusion of even 1-2 $U$ of packed red blood cells was associated with increased 30-day mortality, surgical site infection, pneumonia and sepsis in general surgery patients, whereas others declared that only a large volume of BTF increased the risk (Bellantone et al. 1998; Bernard et al. 2009; Ferraris et al. 2012; Xiao et al. 2014). In the present study, only patients who were given a high-volume (> 7.5 U) BTF showed an increased risk of infection, while administration of a low and moderate volume showed no significant impact. It should be borne in mind that patients who required large-volume BTF, always suffer from severe anemia or massive bleeding, meaning a worse physical condition and a higher risk of post-operative complications.

Allogeneic leukocytes are assumed to play a critical role in inducing TRIM and significantly affect the post-operative outcomes in various types of surgery (Cervia et al. 2007; Tartter et al. 1998). There has been a randomized trial concluded that both the incidence of the operative site and nosocomial infections were significantly higher in patients transfused with packed red cells than those in patients transfused with leukocyte-depleted red cells; but there were only 13 stomach procedures (Tartter et al. 1998). In our hospital, patients who were given BTF received red blood cells without leukocyte depletion before 2014, with depletion after then, giving us the opportunity to evaluate the impact of leukocyte depletion on the incidence of infection directly. As shown in Table 4c, patients who received a leukocyte non-depleted BTF had a significantly higher risk of developing an infection compared to those not receiving a BTF (13.6 vs $6.9 \%, P=0.018$ ), while receiving leukocyte-depleted BTF seemed not to increase the risk $(10.9 \%, P=0.097)$. The present study supports the hypothesis that susceptibility to infection following BTF is due to leukocytes in the transfused blood, and clearly supports the use of leukocytedepleted blood in patients undergoing gastrectomy for gastric caner who require a BTF.

In consistent with our previous study and similar studies from other institutes, a longer operative time ( $\geq 240 \mathrm{~min}$ ), combined multi-organ resection and being overweight (BMI $\geq 25 \mathrm{~kg} / \mathrm{m}^{2}$ ) were identified as independent risk factors for post-operative infection following gastrectomy for gastric cancer (Xiao et al. 2017; Brar et al. 2012; Hirao et al. 2013; Lee et al. 2014). It is easy to understand why and has been discussed in our previous study (Xiao et al. 2017). In contrast to the conclusions of the present investigation, peri-operative $\mathrm{BTF}$ was not identified as an independent risk factor for intra-abdominal infections (IAI) in our previous study. While BTF has been identified as an independent risk factor for pneumonia and sepsis after upper gastrointestinal cancer resections in several previous studies (Aquina et al. 2015; Miki et al. 2016). To explore the underlining reasons, we further divided infections into local infections (including IAI and wound infection) and systemic infections (including pulmonary infection, sepsis and urinary tract infection). Among the matched 361 patients who were not given BTF, 25 patients developed post-operative infection, including 18 cases of local infections and 9 cases of systemic infections (2 patients developed both local and systemic infections); while in the 43 patients who developed infection among the 361 patients receiving BTF, there were 22 cases of local and 24 cases of systemic infections ( 3 patients developed both local infections and systemic infections). The incidence of local infections were similar in the patients receiving BTF or did not (6.1 vs $5.0 \%, P=0.515$ ), but systemic infection was significantly more common in the BTF group compared to the non-BTF group of patients (6.6 vs $2.5 \%, P=0.008$ ). Thus, BTF may mainly increase the risk of systemic infection but has a limited influence on the occurrence of local infection. A possible explanation is that local infections are largely dependent on surgical procedures, whereas systemic infections are mainly associated with systemic inflammation and immunity, but the exact underlying mechanisms remain unclear.

As reported in other studies, peri-operative BTF was clarified as a predictor for infection by multivariate analysis after matching in the present study (Squires et al. 2015; Jung 
et al. 2013; Brar et al. 2012). By carefully reviewing the medical records, we found that there were eight patients who received BTF simultaneously or after infection, but without any clinical evidence of bleeding. A low-hemoglobin level and hemodilution, as a result of extra-cellular expansion during the stress response in patients who developed severe infections, may act as a confounder for the association between BTF and the complications of infection (Bellantone et al. 1998). As listed in Table 3, 43 patients (11.9\%) who received BTF developed an infection, which was significantly more common than in those not receiving a BTF ( 25 cases, $6.9 \%, P=0.022$ ). But if we ruled out the eight pairs of patients, the incidence of infection was comparable between the patients receiving BTF or not $(9.9$ vs $7.1 \%$, $P=0.177)$. Maybe it is not BTF itself but the circumstances necessitating BTF that are the real determinants of outcomes (Bellantone et al. 1998), although a prospective international multi-center study with a larger sample size will be needed to confirm this conjecture.

There are several limitations of the present study including its retrospective nature, single-institution design and insufficient data on immune functions. In addition, some patients in the present study received platelet or plasma transfusions, which may also affect the patients' immune status or interact with BTF to influence post-operative outcomes; we did not investigate these potential associations (Xie et al. 2015; Subramanian et al. 2012). Last but not the least, although propensity score-matching analysis has the advantage of reducing selective bias, it restricts the analysis to a relatively small proportion of the patients, thus dramatically increases the possibility of a type II error and limits the statistical power. However, the prospectively registered highvolume sample database that collected and stored detailed data, the combination use of propensity score matching and multivariate analyses can offer more powerful statistical strength and make our final conclusions to be more robust and reliable. Given it would be unethical to randomize patients to perform BTF or not, large sample-based observational analysis is the best alternative to investigate the effects of BTF on the post-operative infection rate.

In conclusion, the propensity score-matched analysis from a high-volume center in China revealed that infection was the most common complication following gastrectomy for gastric cancer, leading to prolonged hospitalization, a higher frequency of requiring intensive care and also mortality. Peri-operative BTF was identified as an independent risk factor for developing infection, especially a systemic infection such as pneumonia. Patients who were given high-volume ( $>7.5 \mathrm{U}$ ) leukocyte non-depleted BTF and transfused intra-operatively seemed to be at the highest risk. But it should be remembered that the real relationship between BTF and infection maybe confused by the chronological order and further prospective studies are definitely warranted.

Acknowledgements This study was funded by the 2017 Annual Research Project of Health and Family Planning Commission of Hunan Province to Xiao Hua (No. B2017101).

\section{Compliance with ethical standards}

Conflict of interest The authors declare that they have no conflict of interest.

Ethical approval All procedures performed in studies involving human participants were in accordance with the ethical standards of the institutional and/or national research committee and with the 1964 Helsinki declaration and its later amendments or comparable ethical standards.

Open Access This article is distributed under the terms of the Creative Commons Attribution 4.0 International License (http://creativeco mmons.org/licenses/by/4.0/), which permits unrestricted use, distribution, and reproduction in any medium, provided you give appropriate credit to the original author(s) and the source, provide a link to the Creative Commons license, and indicate if changes were made.

\section{References}

Ahmad R, Schmidt BH, Rattner DW, Mullen JT (2014) Factors influencing readmission after curative gastrectomy for gastric cancer. J Am Coll Surg 218:1215-1222

Ajani JA, D'Amico TA, Almhanna K et al (2016) Gastric cancer, version 3. 2016, NCCN Clinical practice guidelines in oncology. $\mathrm{J}$ Natl Compr Canc Netw 14:1286-1312

American Society of Anesthesiologists Task Force on Perioperative Blood Transfusion and Adjuvant Therapies (2006) Practice guidelines for perioperative blood transfusion and adjuvant therapies: an updated report by the American Society of Anesthesiologists Task Force on Perioperative Blood Transfusion and Adjuvant Therapies. Anesthesiology 105:198-208

Aquina CT, Blumberg N, Probst CP et al (2015) Significant variation in blood transfusion practice persists following upper GI cancer resection. J Gastrointestinal Surg 19:1927-1937

Aquina CT, Blumberg N, Becerra AZ et al (2017) Association among blood transfusion, sepsis, and decreased long-term survival after colon cancer resection. Ann Surg 266:311-317

Bellantone R, Sitges-Serra A, Bossola M et al (1998) Transfusion timing and postoperative septic complications after gastric cancer surgery: a retrospective study of 179 consecutive patients. Arch Surg 133:988-992

Bernard AC, Davenport DL, Chang PK et al (2009) Intraoperative transfusion of $1 \mathrm{U}$ to $2 \mathrm{U}$ packed red blood cells is associated with increased 30-day mortality, surgical-site infection, pneumonia, and sepsis in general surgery patients. J Am Coll Surg 208:931-937

Birgegård G, Aapro MS, Bokemeyer C et al (2005) Cancer-related anemia: pathogenesis, prevalence and treatment. Oncology 68(Suppl 1):3-11

Brar SS, Seevaratnam R, Cardoso R et al (2012) Multivisceral resection for gastric cancer: a systematic review. Gastric Cancer 15(Suppl 1):S100-107 
Cervia JS, Wenz B, Ortolano GA (2007) Leukocyte reduction's role in the attenuation of infection risks among transfusion recipients. Clin Infect Dis 45:1008-1013

Chen W, Zheng R, Baade PD et al (2016) Cancer statistics in China, 2015. CA Cancer J Clin 66:115-132

Cunningham D, Allum WH, Stenning SP et al (2006) Perioperative chemotherapy versus surgery alone for resectable gastroesophageal cancer. N Engl J Med 355:11-20

Dindo D, Demartines N, Clavien PA (2004) Classification of surgical complications: a new proposal with evaluation in a cohort of 6336 patients and results of a survey. Ann Surg 240:205-213

Ecker BL, Simmons KD, Zaheer S et al (2016) Blood transfusion in major abdominal surgery for malignant tumors: a trend analysis using the national surgical quality improvement program. JAMA Surg 151:518-525

Elmi M, Mahar A, Kagedan D et al (2016) The impact of blood transfusion on perioperative outcomes following gastric cancer resection: an analysis of the American College of Surgeons National Surgical Quality Improvement Program database. Can J Surg 59:322-329

Ferraris VA, Davenport DL, Saha SP et al (2012) Surgical outcomes and transfusion of minimal amounts of blood in the operating room. Arch Surg 147:49-55

Fujiya K, Tokunaga M, Mori K et al (2016) Long-term survival in patients with postoperative intra-abdominal infectious complications after curative gastrectomy for gastric cancer: a propensity score matching analysis. Ann Surg Oncol 23:809-816

Hirao M, Tsujinaka T, Imamura $\mathrm{H}$ et al (2013) Overweight is a risk factor for surgical site infection following distal gastrectomy for gastric cancer. Gastric Cancer 16:239-244

Horan TC, Andrus M (2008) CDC/NHSN surveillance definition of health care-associated infection and criteria for specific types of infections in the acute care setting. Am J Infect Control 36:309-332

Japanese Gastric Cancer Association (2017) Japanese gastric cancer treatment guidelines 2014 (ver. 4). Gastric Cancer 20:1-19

Jung DH, Lee HJ, Han DS et al (2013) Impact of perioperative hemoglobin levels on postoperative outcomes in gastric cancer surgery. Gastric Cancer 16:377-382

Kanda M, Kobayashi D, Tanaka C et al (2016) Adverse prognostic impact of perioperative allogeneic transfusion on patients with stage II/III gastric cancer. Gastric Cancer 19:255-263

Kwon SJ (2011) Evaluation of the 7th UICC TNM staging system of gastric cancer. J Gastric Cancer 11:78-85

Lee KG, Lee HJ, Yang JY et al (2014) Risk factors associated with complication following gastrectomy for gastric cancer: retrospective analysis of prospectively collected data based on the Clavien-Dindo system. J Gastrointest Surg 18:1269-1277

Miki Y, Makuuchi R, Tokunaga M et al (2016) Risk factors for postoperative pneumonia after gastrectomy for gastric cancer. Surg Today 46:552-556

Rubin DB, Thomas N (1996) Matching using estimated propensity scores: relating theory to practice. Biometrics 52:249-264

Sasako M, Sano T, Yamamoto S et al (2008) D2 lymphadenectomy alone or with para-aortic nodal dissection for gastric cancer. $\mathrm{N}$ Engl J Med 359:453-462

Smyth EC, Verheij M, Allum W et al (2016) Gastric cancer: ESMO clinical practice guidelines for diagnosis, treatment and follow-up. Ann Oncol 27:v38-v49

Squires MH III, Kooby DA, Poultsides GA et al (2015) Effect of perioperative transfusion on recurrence and survival after gastric cancer resection: a 7-institution analysis of 765 Patients from the US Gastric Cancer Collaborative. J Am Coll Surg 211:767-777

Subramanian A, Berbari EF, Brown MJ et al (2012) Plasma transfusion is associated with postoperative infectious complications following esophageal resection surgery: a retrospective cohort study. J Cardiothorac Vasc Anesth 26:569-574

Sun C, Wang Y, Yao HS, Hu ZQ (2015) Allogeneic blood transfusion and the prognosis of gastric cancer patients: systematic review and meta-analysis. Int J Surg 13:102-110

Tartter PI, Mohandas K, Azar P et al (1998) Randomized trial comparing packed red cell blood transfusion with and without leukocyte depletion for gastrointestinal surgery. Am J Surg 176:462-466

Torre LA, Bray F, Siegel RL et al (2015) Global Cancer Statistics 2012. CA Cancer J Clin 65:87-108

Xiao H, Ouyang Y, Tang M et al (2014) Association of perioperative and postoperative complications after radical gastrectomy for gastric cancer. Zhonghua Yi Xue Za Zhi 94:751-754 (Article Chinese)

Xiao H, Xiao Y, Quan H et al (2017) Intra-abdominal infection after radical gastrectomy for gastric cancer: incidence, pathogens risk factors and outcomes. Int J Surg 48:195-200

Xie RF, Hu P, Wang ZC et al (2015) Platelet-derived microparticles induce polymorphonuclear leukocyte-mediated damage of human pulmonary microvascular endothelial cells. Transfusion 55:1051-1057

Yang T, Lu JH, Lau WY, Zhang TY, Zhang H, Shen YN et al (2016) Perioprioperative blood transfusion does non influence recurrence-free and overall survival after curative resection for hepatocellular carcinoma: a propensity score matching analysis. J Hepatol 64:583-593 